\title{
Cirurgia em tempos de COVID-19
}

\author{
Humberto Ferraz ${ }^{1}$
}

1 Professor de cirurgia da Universidade do Estado da Bahia (UNEB), com mestrado em Medicina e Saúde Humana Escola Bahiana de Medicina e Saúde Pública (EBMSP). É doutorando pelo Programa de Pós-Graduação em Ciências Sociais (PPGCS) da Universidade Federal da Bahia (UFBA) e médico urologista do Hospital Santa Izabel (Santa Casa de Misericórdia da Bahia).

FERRAZ, H. Cirurgia em tempos de COVID-19. In: BARRETO, M.L.; PINTO JUNIOR, E. P.; ARAGÃO, E.; BARRAL-NETTO, M. (org.). Construção de conhecimento no curso da pandemia de COVID-19: aspectos biomédicos, clínico-assistenciais, epidemiológicos e sociais. Salvador: Edufba, 2020. v. 2. DOI: https://doi.org/10.9771/9786556300757.017 


\section{Introdução}

O ambiente de centro cirúrgico foi historicamente preparado para prevenção de infecção por agentes transmitidos através de contato com sangue e secreções principalmente. Equipamentos de Proteção Individual (EPIs) e para a equipe, cuidados com os pacientes, limpeza de ambientes e materiais após os procedimentos foram direcionados para evitar contaminação por bactérias multirresistentes ou vírus transmitidos através do contato direto com pele e mucosa. Entretanto, a proteção para aerossóis não fazia parte dessa rotina. Os centros cirúrgicos são estruturados em uma área fechada, com pouca troca de ar e, geralmente, sem pressão negativa. Adicionalmente, a maioria dos pacientes anestesiados fazem uso de ventilação mecânica. Essas condições favorecem a transmissão de infecção pelo novo coronavírus entre os pacientes, membros da equipe de cirurgia e funcionários do setor.

Frente à necessidade de mudanças, para tornar cirurgias e procedimentos mais seguros durante a pandemia, surgiram publicações de instituições de prestígio na comunidade médica, com novas rotinas a serem consideradas até a concretização de estudos científicos com maior nível de evidencia. Recente revisão sistemática 
de Moletta e demais autores (2020) em publicações de língua inglesa, utilizando as palavras-chave: "pandemic", "coronavirus”, “COVID-19”, e "surgery" ou "surgical”, após levantamento inicial de 1076 artigos, foram elegíveis para avaliação 22 papers (13 guidelines e nove artigos originais). Foram destacados:

1. necessidade de abordagem de todos os pacientes como potencialmente infectados, com medidas de proteção individual e coletiva;

2. critérios para priorização de casos eletivos;

3. importância de assegurar o tratamento cirúrgico em caráter de urgência e emergência;

4. opção por tratamentos não cirúrgicos sempre que possível. (MOLETTA et al., 2020)

Esses e outros tópicos serão detalhados a seguir.

\section{Qual a frequência e riscos de contaminação entre equipe e pacientes cirúrgicos?}

Há vários relatos de infecção por SARS-CoV-2 detectada no pós-operatório e a infecção por SARS-CoV em pacientes cirúrgicos parece aumentar a morbimortalidade. (AMINIAN et al., 2020) Estudo de coorte multicêntrico envolvendo 1128 pacientes que desenvolveram infecção pelo SARS-CoV-2 no período perioperatorio, compreendendo período entre sete dias pré-operatório e 30 dias no pós-operatório, foi encontrado elevada frequência de complicações respiratórias $(51,2 \%)$ e mortalidade de $38 \%$ nesse grupo. (COLLABORATIVE, 2020b) O risco foi maior em homens, idade superior a 70 anos, cirurgia de urgência, risco anestésico na escala American Society of Anesthesiologists (ASA) 3 a 5, portadores de 
malignidade e em cirurgias de grande porte. Quando avaliada apenas cirurgias eletivas, houve uma taxa de mortalidade de 20,4\% entre os que desenvolveram COVID-19 no período pós-cirúrgico. Ainda não há estudos determinando a taxa de infecção intra-hospitalar por SARS-CoV-2 em pacientes cirúrgicos.

Entretanto, a taxa de contaminação durante a estadia no hospital entre os profissionais de saúde tem se mostrado elevada em diversos estudos. Na Europa, há relatos de cerca de um quinto de infectados entre os profissionais de saúde que trabalham no enfrentamento da primeira onda da epidemia. Na Itália, em torno de $20 \%$ dos profissionais de saúde apresentaram sintomas relacionados ao SARS-CoV-2. (REMUZZI; REMUZZI, 2020) A mesma taxa de afastamento do trabalho foi observada em um inquérito feito pelo Royal College of Physicians, em abril, com 2513 profissionais - 21,5\% em Londres e 18,3\% no restante da Inglaterra. (MAYOR, 2020)

Não há estudos até o momento mostrando a taxa de infecção entre os trabalhadores do centro cirúrgico. Entretanto, o aumento na demanda e o afastamento de profissionais de saúde envolvidos no enfrentamento direto da epidemia podem desfalcar as equipes que realizam atividades cirúrgicas.

\section{Quais os equipamentos individuais essenciais para a proteção dos profissionais no centro cirúrgico?}

Considerando a situação de transmissão comunitária do vírus, como ocorre no Brasil e a confirmação de transmissão de COVID-19 por assintomáticos, tem sido recomendado o uso rotineiro de EPIs com proteção para contaminação por aerossóis dentro do centro cirúrgico. 
A nota técnica da Gerência de Vigilância e Monitoramento em Serviços de Saúde (GVIMS) e da Gerência Geral de Tecnologia em Serviços de Saúde (GGTES) da Agência Nacional de Vigilância Sanitária (Anvisa) $n^{\circ}$ 06/2020 destaca como essencial o uso de máscara N95, protetor facial (face shield), óculos de proteção, gorro e roupa impermeável (LIVINGSTON, 2020), estando de acordo com as recomendações do Centers for Disease Control (CDC).

Há risco de contaminação principalmente relacionada ao mal uso e a retirada dos EPIs (desparamentação). Técnicas de treinamento da equipe e supervisão por outro profissional no momento da colocação e retirada desses EPIs têm sido preconizadas. (BRAT et al., 2020; BRINDLE; GAWANDE 2020) Para diminuir esse risco, é importante seguir a sequência recomendada de higienização das mãos diversas vezes nesse processo, retirada de EPI dentro e fora da sala de cirurgia, além de banho e troca de roupa privativa. (ONG et al., 2020)

\section{Quais os cuidados na intubação orotraqueal para anestesia geral?}

Um dos momentos críticos da contaminação na sala de cirurgia é durante a intubação orotraqueal. Para minimizar o risco para o anestesista e equipe cirúrgica, alguns protocolos foram desenvolvidos. Para proteção do anestesista, tem sido preconizada mudança na rotina de indução, que é o momento em que se inicia a anestesia. As medidas visam modificar o tempo e o tipo de contato do anestesista com o paciente. Para encurtar o momento da indução, sugere-se a redução no tempo de ventilação prévia à intubação e sedação/curarização em sequência rápida. (BREWSTER et al., 2020; COOK et al., 2020; KOVACS et al., 2020) Além disso, durante a intubação, o distanciamento entre 
o anestesista e o paciente tem sido realizado com uso de videolaringoscópio para guiar a intubação, e de caixas de acrílico e sacos plásticos. (CANELLI et al., 2020)

Outra estratégia é reduzir número de pessoas expostas à contaminação na sala de cirurgia, especificamente no momento da intubação e extubação, quando são dispersadas mais partículas de aerossóis. Há recomendação para que o cirurgião e sua equipe aguardem dez minutos após a intubação para entrar na sala operatória. Da mesma forma, sugerem que a equipe de cirurgia retire-se da sala antes da extubação do paciente. (KIM et al., 2020)

\section{Quais as orientações sobre testagem diagnóstica para equipe e pacientes e para o afastamento de profissionais?}

O exame de escolha para deteção de indivíduos - pacientes ou equipe - em fase de potencial contaminação é a Reverse Transcription Polymerase Chain Reaction (RT-PCR). Visando à deteção de infecção assintomática, tem sido proposta a testagem de todos os envolvidos. A testagem do paciente eletivo tem sido preconizada pelo Colégio Brasileiro dos Cirurgiões (CBC), geralmente dois dias antes do procedimento, para reduzir o risco de contaminação da equipe e outros contatantes, além de minimizar o risco de complicações pos operatórias. Entretanto, não descarta totalmente o risco de contaminação, considerando que pacientes podem infectar-se entre a coleta da amostra e a realização da cirurgia. Adicionalmente, vale ressaltar que o teste de RT-PCR pode ter resultado falso-negativo por apresentar sensibilidade relativamente baixa. (XIAO et al., 2020)

Quanto à testagem de membros da equipe, não há no momento um consenso sobre o número e intervalo das avaliações de assintomáticos. Obviamente quanto maior a frequência de testagem, maior a possibilidade de deteç̧ão. O fator limitante 
costuma ser o custo e carência de insumos para testes rotineiros e em massa em toda equipe.

Para vigilância de infecção, os hospitais tem indicado o rastreamento de sintomáticos e o afastamento de todos os contatantes de pessoas com COVID-19. (LIANG et al., 2020) Para minimizar o impacto do afastamento de membros da equipe de saúde, algumas estratégias têm sido adotadas, como divisão de grandes grupos que passam a trabalhar em pequenas equipes fixas, expondo apenas uma parte dos profissionais a cada cirurgia e possibilitando um rodízio de grupos em caso de afastamento por contaminação de um dos membros.

Sobre o retorno às atividades do profissional de saúde após infecção pelo SARS-CoV-2, são recomendadas estratégias dependentes ou independentes de testagem. Quando testes forem utilizados, além de ausência de sintomas respiratórios e febre, o profissional deve apresentar pelo menos um RT PCR com resultado negativo. Sem o uso do teste, o profissional deve ter pelo menos 72 horas sem sintomas e no mínimo 14 dias desde o início da doença ou teste inicial positivo. Alguns locais onde ocorreram escassez de profissionais de saúde tiveram esse período de afastamento reduzido para sete dias. (CENTERS FOR DISEASE CONTROL AND PREVENTION , 2020b; DIAS, 2020; FERRETTI et al., 2020)

\section{Cirurgias eletivas devem ser realizadas durante a pandemia?}

A rotina de cirurgias eletivas sofreu abrupta interrupção desde início da pandemia. (COLLABORATIVE, 2020a; HORTON, 2020) Em curva ascendente de número de casos e internações pela COVID-19, infecção no período perioperatório e o risco de desenvolver COVID-19 no pós-operatório tem sido reportados. (AMINIAN et al., 
2020) Surgiram recomendações de reprogramação de cirurgias eletivas e diversas especialidades cirúrgicas fizeram propostas de tempo de postergação dos procedimentos conforme possibilidade de evolução da doença, sequelas permanentes e risco de abreviar mortalidade. (ACS, 2020)

Há dois tipos de cirurgia eletiva: opcional - por exemplo, estética - e não opcional - como a oncológica. A postergação de cirurgias eletivas não opcional terá consequências danosas à saúde pública. Há preocupação em especial em relação às cirurgias oncológicas. Análise realizada na Inglaterra estima que a postergação de cirurgia oncológica por seis meses aumentará cerca de $30 \%$ na mortalidade de cinco anos de pacientes oncológicos de diferentes idades, sítios e estágios 1, 2 ou 3 da doença. Em números absolutos, representam 4700 mortes por ano atribuídas a um retardo de três a seis meses na realização do procedimento cirúrgico. (BARTLETT et al., 2020; SUD et al., 2020) As estimativas variam conforme o tipo de tumor. Para neoplasia de próstata, por exemplo, um estudo realizado por Ginsburg e demais autores (2020), baseado em regressão logística multivariada de casos tratados entre 2010 e 2016, estima que não haverá aumento da mortalidade em candidatos à prostatectomia radical com cirurgia postergada por até 12 meses. Nesse mesmo estudo, o retardo na cirurgia em grupos histológicos mais indiferenciados - grupos 4 e 5 de Gleason - resultou em piora no prognóstico. (GINSBURG et al., 2020; HELDWEIN et al., 2020) A análise desses dados pode orientar estratégias de priorização dos casos na retomada das cirurgias eletivas e mitigar as mortes atribuídas à postergação do tratamento. Estratégias semelhantes estão sendo adotadas por radioterapeutas com intuito de reduzir complicações e internações. (CHEN et al., 2020) A priorização dos casos oncológicos deve considerar risco individual de uma possível exposição à COVID-19 e alternativas ao tratamento cirúrgico. Nesse sentido, 
a American Society of Clinical Oncology (ASCO) e outras sociedades de oncologia sugerem reuniões multidisciplinares para decisão da melhor opção terapêutica, considerando, para a tomada da decisão, o momento da pandemia e os recursos disponíveis (UTI, equipe e materiais). Essas decisões apoiadas na opinião de vários especialistas poderão ser úteis também após o declínio da curva da epidemia e o retorno do atendimento em consultórios e ambulatórios, quando se estima uma maior frequência de diagnósticos oncológicos com estadiamento avançado.

Além das cirurgias oncológicas, outros tipos de cirurgias eletivas necessárias incluem procedimentos para alívio de dor, para melhora de função de órgãos e da qualidade de vida e para prevenção de complicações ou progressão de doença com sequelas permanentes como amputação de membros.

As definições da conduta passarão por uma decisão compartilhada entre cirurgião e paciente, incluindo discussão sobre resultado de tratamentos alternativos, adiamento da cirurgia, riscos de transmissão de SARS-CoV-2 para o time cirúrgico, de contrair o vírus durante estadia hospitalar e das complicações relacionadas à COVID-19 no pós-operatório. Para dar suporte a essas decisões, alguns escores têm sido propostos por sociedades de especialidades e serviços de referência, ainda sem validação externa. O departamento de cirurgia da Universidade de Chicago propôs o escore Medically Necessary Time Sensitive (MeNTS), utilizando pontuação para o procedimento, o perfil do paciente e a doença, podendo variar de 25 a 105 pontos. (PRACHAND et al., 2020) Quanto menor a pontuação, mais seguro e necessário seria o procedimento, com potencial de aplicação em hospitais de perfis diferentes, acadêmicos ou não. 


\section{Quais os cuidados com as cirurgias de urgência?}

Milhares de pacientes são operados diariamente durante a pandemia ainda que os procedimentos eletivos tenham sido postergados, considerando a frequência de procedimentos cirúrgicos de urgência realizados usualmente. (GALE et al., 2014) Os critérios de determinação da urgência de cada caso independem da pandemia e deve ser dada a atenção adequada para não ocorrer retardo no procedimento e consequente pior evolução do quadro. (COCCOLINI et al., 2020b) Assim, tem sido discutido o cuidado necessário para realização desses procedimentos com segurança.

De acordo com Coimbra e demais autores (2020), experiências multicêntricas de serviços de emergência, respaldadas pela European Society of Emergency Surgery, indicam cuidados divididos em oito domínios principais:

1. recomendações gerais para serviços de cirurgia;

2. preparo pré-operatório;

3. seleção de casos para cirurgia de urgência em pacientes COVID-19 positivos ou suspeitos;

4. preparo da sala de cirurgia;

5. transporte do paciente para o centro cirúrgico;

6. preparação da equipe cirúrgica;

7. considerações anestésicas; e

8. conclusão com revisão do caso. (COIMBRA et al., 2020)

De acordo com os autores, com impossibilidade temporal de realizar PCR antes da cirurgia em pacientes com indicação de urgência, a princípio todos os pacientes devem ser considerados como potenciais portadores de COVID-19. Sugere exames de imagem do tórax para fins de avaliação diagnóstica, destacando-se a tomografia como exame mais acurado nessa situação. (AI et al., 2020) Em investigação de dor abdominal, sugere realizar 
Tomografia Computadorizada (TC) do tórax quando for realizar a TC de abdômen, pois COVID-19 tem-se apresentado com sintomas gastrointestinais simulando abdome agudo. (ROYAL COLLEGE OF SURGEONS OF EDINBURGH et al., 2020; TIAN et al., 2020)

\section{Qual o ambiente ideal para cirurgia de pacientes com GOVID-19?}

A cirurgia de um paciente com infecção confirmada por COVID-19 envolve mudanças no transporte até o centro e dentro dessa unidade. Estudos mostraram a identificação de presença do vírus em suspensão e em superfície de salas e equipamentos isolados para cirurgias em portadores de COVID-19 (ONG et al., 2020, VAN DOREMALEN et al., 2020). Medidas são sugeridas para evitar contato com outros pacientes no percurso até o centro cirúrgico e a liberação de aerossóis no trajeto. Dentro do centro cirúrgico, tem sido sugerida a reserva de ambiente especifico para cirurgia desses pacientes, com redução de mobiliário e equipamentos, envelopamento de matérias, redução do tamanho da equipe cirúrgica e manutenção de um sistema de pressão negativa, com troca frequente de ar. (CDC, 2020; CORREIA, 2020; BRINDLE; GAWANDE, 2020; FORRESTER et al., 2020; TI et al., 2020; WONG et al., 2020)

\section{Existem procedimentos com maior risco de transmissão?}

O vírus está presente em diversos tecidos e fluidos corporais. Partículas virais foram encontradas em $s w a b$ nasal, faringe, escarro, secreção brônquica, tecido gastrointestinal, sangue, fezes e fluido peritoneal. (COCCOLINI, 2020a; WANG, 2020) Dessa forma, existem procedimentos com maior risco, mas todo procedimento cirúrgico 
deve ser considerado um momento potencial para contaminação. (BRAT et al., 2020)

As cirurgias em vias aéreas são consideradas de risco elevado de disseminação de SARS-CoV-2 por ser local de alta carga viral. Em consequência, especialidades que fazem cirurgias nesses campos tais como odontólogos, otorrinolaringologistas, cirurgia de cabeça e pescoço e cirurgia torácica estão sob maior risco de infecção. (COULOIGNER et al., 2020; FORRESTER et al., 2020)

Algumas particularidades são importantes no cenário do centro cirúrgico. Uma delas é a cirurgia videolaparoscópica assistida ou não por robô -, que tem como fundamento a criação de um espaço intracavitário, peritoneal ou extraperitoneal, com insuflação do CO2. (ZHENG et al., 2020) Esse gás mistura-se com o fluido abdominal, com fumaça e partículas geradas pelas diferentes formas de energia utilizadas para diérese e cauterização de tecidos (bisturi elétrico, energia ultrassônica). O gás pode escapar para a sala cirúrgica e precisa ser evacuado parcialmente, em alguns momentos do intraoperatório, ou completamente, no final da cirurgia. Presença ativa do Vírus da Imunodeficiência Humana (HIV), Papilomavírus Humano (HPV) e Corynebacterium foi detectada nessa fumaça, o que leva a preocupação da possibilidade de transmissão do SARS-CoV-2 por essa via. (CAPIZZI; CLAY; BATTEY, 1998; GLOSTER; ROENIGK, 1995; JOHNSON; ROBINSON, 1991; HENSMAN et al., 1998) Corroborando essa hipótese, o SARS-CoV-2 foi isolado no fluido peritoneal de pacientes sintomáticos submetidos à cirurgia de urgência. Medidas têm sido consideradas para minimizar escape do $\mathrm{CO} 2$ durante o ato operatório: aplicar baixa pressão do gás sempre que possível, utilizar equipamentos para melhorar a vedação - borrachas, trocateres - e realizar a filtragem do ar. Semelhante cuidado com filtragem do ar deve ser adotado com o aspirador utilizado na cirurgia. 
O uso de bisturi elétrico e ultrassônico tem sido apontado como fonte de geração de aerossóis e possivel disseminação do virus. (LI; PAI; CHEN, 2020) Nesse sentido, há recomendações de uso preferencial de fios, clips metálicos e de polímeros para hemostasia.

\section{O que fazer para o retorno seguro dos procedimentos cirúrgicos após o surgimento do SARS-CoV-2 ?}

A circulação do SARS-CoV-2 deverá persistir por pelo menos alguns anos. Considerando a taxa de soroconversão nas populações que sofreram primeiro com a epidemia e as obtidas nos primeiros estudos no Brasil (HALLAL et al., 2020a, 2020b), há riscos de surgimento de novas ondas. Solução mais definitiva, como a vacina, ainda que desenvolvida nos próximos 12 meses, levará longo tempo até ser produzida e aplicada em larga escala. (ROWLAND; JOHNSON; WAN, 2020) Assim, estratégias para retomar as rotinas de cirurgia, reduzindo a fragilidade do sistema de saúde, de forma a conviver com novas ondas de infecção pelo coronavírus, são necessárias.

A monitorização e utilização de um sistema de informação confiável da situação epidemiológica local pode contribuir para a segurança de profissionais e pacientes. (COBIANCHI et al., 2020)

O American College of Surgeons tem preconizado atenção aos hospitais quanto a estruturação antes de liberação de cirurgias eletivas, destacando o cumprimento de guidelines, o acompanhamento com especialistas e lideres locais e nacionais, a avaliação de recursos institucionais e a conformidade com situação epidemiológica e necessidades regionais. (DIAZ et al., 2020) 
Liang e demais autores (2020) pontuam características das atividades cirúrgicas nas eras pré e pós-COVID-19. No período de resiliência, com o surgimento do vírus, foram expostas as fragilidades dos serviços para lidar com a pandemia de COVID-19 e houve a necessidade de ajustar a realidade hospitalar a novos protocolos de segurança. Além disso, houve redimensionamento de demanda cirúrgica eletiva e disponibilização de recursos e leitos para COVID-19. Os autores sugerem a adoção de sete passos para um retorno seguro das cirurgias eletivas. Conhecer em que ponto se encontra na curva epidêmica; garantir testagem segura e acessível preferencialmente com RT-PCR; garantir recursos hospitalares disponíveis sem comprometer assistência a urgências; discutir seleção de casos e critérios de priorização; otimizar o plano de recuperação do paciente após a alta; retomar casos de forma gradual; e estar preparado para possíveis novos problemas e interrupções. (LIANG et al., 2020)

A aplicação de telemedicina tem sido proposta como solução para o acompanhamento pós-operatório, reduzindo o retorno aos serviços de saúde para reavaliação e minimizando o risco de infecção nos pacientes convalescentes de cirurgia. (COLÉGIO BRASILEIRO DE CIRURGIÕES, 2020; SMITH et al., 2020; SORENSEN et al., 2020)

\section{Considerações finais}

Dado o exposto, a pandemia da COVID-19 causou importante impacto na dinâmica de realização de cirurgias em todo o mundo. O redirecionamento dos recursos humanos, espaços físico e insumos para atendimento às demandas relacionadas à pandemia, além do afastamento de profissionais de saúde acometidos pela doença, forçou a interrupção das programações 
eletivas e retardou procedimentos de urgência. Ações de mitigação do impacto da pandemia na área cirúrgica visam, em primeiro momento, priorizar cirurgias essenciais e com possibilidade de sequelas definitivas, seguindo protocolos institucionais para segurança de pacientes e equipe cirúrgica. O retorno à rotina de atividades cirúrgicas deverá ser apoiada na implementação de protocolos de segurança baseados no conhecimento científico sobre a doença e respeitando o momento epidemiológico da epidemia em cada região. Por fim, deve ser estimulada a busca por inovações como uso da telemedicina e outras ferramentas de proteção individual e coletiva que contribuam para maior segurança no contato entre médicos e pacientes.

\section{Referências}

AGÊNCIA NACIONAL DE VIGILÂNCIA SANITÁRIA (Brasil). Nota Técnica GVIMS/GGTES/ANVISA No 06/2020 - Orientações para a prevenção e o controle das infecções pelo novo coronavírus (sars-cov-2) em procedimentos cirúrgicos - 29.05.2020 (Complementar à Nota Técnica GVIMS/GGTES/ANVISA No 04/2020). Brasília, DF, 2020. Disponível em: http://portal.anvisa.gov.br/ documents/33852/271858/Nota+t\%C3\%A9cnica+06-2020+GVIMS-GGTESANVISA/40edaf7d-8f4f-48c9-b876-bee0090d97ae. Acesso em: 14 jun. 2020.

AMERICAN COLLEGE OF SURGEONS - ACS. ACS Guidelines for Triage and Management of Elective Cancer Surgery Cases During the Acute and Recovery Phases of Coronavirus Disease 2019 (COVID-19) Pandemic. [Chicago], 2020. Disponível em: https://www.facs.org/-/media/files/covid19/acs_triage_and_ management_elective_cancer_surgery_during_acute_and_recovery_phases.ashx. Acesso em: 7 maio 2020.

AMINIAN, A. et al. COVID-19 Outbreak and Surgical Practice: Unexpected Fatality in Perioperative Period. Annals of Surgery, Philadelphia, v. 272, n. 1, p. e27-e29, 2020. DOI: 10.1097/SLA.0000000000003925. Disponível em: https://journals.Iww. com/annalsofsurgery/FullText/2020/07000/COVID_19_Outbreak_and_Surgical_ Practice_.12.aspx. Acesso em: Acesso em: 9 jul. 2020. 
Al, T. et al. Correlation of Chest CT and RT-PCR Testing in Coronavirus Disease 2019 (COVID-19) in China: A Report of 1014 Cases. Radiology, Easton, PA, 2020. DOI: 10.1148/radiol.2020200642. Disponível em: https://pubs.rsna.org/ doi/10.1148/radiol.2020200642. Acesso em: 9 jul. 2020.

BARTLETT, D. L. et al. Management of Cancer Surgery Cases During the COVID-19 Pandemic: Considerations. Annals of Surgical Oncology, New York, v. 27, n. 6, p. 1717-1720, 2020. DOI: 10.1245/s10434-020-08461-2. Disponível em: Acesso em: 9 jul. 2020.

BRAT, G. A. et al. Protecting Surgical Teams During the COVID-19 Outbreak: A Narrative Review and Clinical Considerations. Annals of Surgery. Philadelphia, 2020. DOI: 10.1097/SLA.0000000000003926. Disponível em: https://journals. Iww.com/annalsofsurgery/Citation/9000/Protecting_Surgical_Teams_During_the COVID_19.94577.aspx. Acesso em: 9 jul. 2020.

BREWSTER, D. J. et al. Consensus statement: Safe Airway Society principles of airway management and tracheal intubation specific to the COVID-19 adult patient group. The Medical Journal of Australia, Sydney, v. 212, n. 10, p. 472-481, 2020. DOI: 10.5694/mja2.50598. Disponível em: https://onlinelibrary.wiley.com/ doi/full/10.5694/mja2.50598. Acesso em: 9 jul. 2020.

CANELLI, R. et al. Barrier Enclosure during Endotracheal Intubation. The New England Journal of Medicine, Boston, v. 382, n. 20, p. 1957-1958, 2020. Disponível em: https://www.nejm.org/doi/10.1056/NEJMc2007589. Acesso em: 9 jul. 2020.

CAPIZZI, P. J.; CLAY, R. P.; BATTEY, M. J. Microbiologic activity in laser resurfacing plume and debris. Lasers in Surgery and Medicine, New York, v. 23, n. 3, p. 172-174, 1998. DOI: 10.1002/(sici)1096-9101(1998)23:3<172: :aid-Ism7>3.0.co;2-m. Disponível em: https://onlinelibrary.wiley.com/doi/abs /10.1002/\%28SICI\%291096-9101\%281998\%2923\%3A3\%3C172\%3A\%3AAIDLSM7\%3E3.0.CO\%3B2-M. Acesso em: 9 jul. 2020.

COLÉGIO BRASILEIRO DE CIRURGIÕES - CBC. Orientações para o retorno de cirurgias eletivas durante a pandemia de COVID-19, maio 2020. Disponível em: https://cbc.org.br/wp-content/uploads/2020/05/PROPOSTA-DE-RETOMADADAS-CIRURGIAS-ELETIVAS-30.04.2020-REVISTO-CBCAMIBSBASBOT-ABIH-SBI-EDEMAIS.pdf. Acesso em: 9 jul. 2020.

CENTERS FOR DISEASE CONTROL AND PREVENTION - CDC. Cases in the U.S. [S. I.], 2020a. Disponível em: https://www.cdc.gov/coronavirus/2019-ncov/casesupdates/summary.html. Acesso em: 9 jul. 2020. 
CENTERS FOR DISEASE CONTROL AND PREVENTION - CDC. Criteria for Return to Work for Healthcare Personnel with SARS-CoV-2 Infection (Interim Guidance). [S. I.], 2020b. Disponível em: https://www.cdc.gov/coronavirus/2019-n-cov/hcp/returnto-work.html. Acesso em: 9 jul. 2020.

CHEN, W. C. et al. Guidelines to Reduce Hospitalization Rates for Patients Receiving Curative-Intent Radiation Therapy During the COVID-19 Pandemic: Report from a Multicenter New York Area Institution. Advances in Radiation Oncology, Philadelphia, 2020. DOI: 10.1016/j.adro.2020.04.021. Disponível em: https://www.advancesradonc.org/article/S2452-1094(20)30097-X/fulltext.. Acesso em: 9 jul. 2020.

COBIANCHI, L. et al. To a New Normal: Surgery and COVID-19 during the Transition Phase. Annals of Surgery, Philadelphia, 2020. DOI: 10.1097/ SLA.0000000000004083. Disponível em: https://journals.Iww.com/annalsofsurgery/ FullText/2020/08000/To_a_New_Normal_Surgery_and_COVID_19_During_the.13. aspx. Acesso em: 9 jul. 2020.

COCCOLINI, F. et al. Surgery in COVID-19 patients: operational directives. World Journal of Emergency Surgery, London, v. 15, n. 25, 2020a. DOI: 10.1186/ s13017-020-00307-2. Disponível em: https://wjes.biomedcentral.com/ articles/10.1186/s13017-020-00307-2. Acesso em: 9 jul. 2020.

COCCOLINI, F. T. et al. SARS-CoV-2 is present in peritoneal fluid in COVID-19 patients. Annals of Surgery, [Philadelphia], 2020b. Disponível em: https://journals. Iww.com/annalsofsurgery/Documents/SARS-CoV-2\%20is\%20present\%20in\%20 peritoneal\%20fluid\%20in\%20COVID-19\%20patients.pdf. Acesso em: 9 jul. 2020.

COIMBRA, R. et al. European Society of Trauma and Emergency Surgery (ESTES) recommendations for trauma and emergency surgery preparation during times of COVID-19 infection. European Journal of Trauma and Emergency Surgery, Munich, v. 46, n. 3, p. 505-510, 2020. DOI: 10.1007/s00068-020-01364-7. Disponível em: https://link.springer.com/article/10.1007\%2Fs00068-020-01364-7. Acesso em: 9 jul. 2020.

COLLABORATIVE, C. O. Elective surgery cancellations due to the COVID-19 pandemic: global predictive modelling to inform surgical recovery plans. The British Journal of Surgery, Bristol, 2020a. DOI: 10.1002/bjs.11746. Disponível em: https://bjssjournals.onlinelibrary.wiley.com/doi/full/10.1002/ bjs.11746. Acesso em: 9 jul. 2020. 
COLLABORATIVE, C. O. Mortality and pulmonary complications in patients undergoing surgery with perioperative SARS-CoV-2 infection: an international cohort study. The Lancet, v. 396, n. 10243, p. 27-38, 2020b. DOI: https://doi. org/10.1016/S0140-6736(20)31182-X. Disponível em: https://www.thelancet.com/ journals/lancet/article/PIIS0140-6736(20)31182-X/fulltext. Acesso em: 9 jul. 2020.

COOK, T. M. et al. Consensus guidelines for managing the airway in patients with COVID-19: Guidelines from the Difficult Airway Society, the Association of Anaesthetists the Intensive Care Society, the Faculty of Intensive Care Medicine and the Royal College of Anaesthetists. Anaesthesia, London, n. 75, n. 6, p. 785-799, 2020. Disponível em: https://onlinelibrary.wiley.com/doi/full/10.1111/ anae.15054. Acesso em: 9 jul. 2020.

CORREIA, M. I. T. D.; RAMOS, R. F.; VON BAHTEN, L. C. Os cirurgiões e a pandemia do COVID- 19. Revista do Colégio Brasileiro de Cirurgiões, Rio de Janeiro, v. 47, n. 1, mar. 2020. https://doi.org/10.1590/0100-6991e-20202536. Disponível em: https:// www.scielo.br/scielo.php?script=sci_arttext\&pid=S0100-69912020000100601\&tlng=pt. Acesso em: 9 jul. 2020.

COULOIGNER, V. et al. COVID-19 and ENT Surgery. European Annals of Otorhinolaryngology. Head and Neck Diseases, [Issy les Moulineaux], v. 137, n. 3, p. 161-166, 2020. DOI: 10.1016/j.anorl.2020.04.012. Disponível em: https:// www.sciencedirect.com/science/article/pii/S1879729620301022?via\%3Dihub. Acesso em: 9 jul. 2020.

DIAS, V. et al. Guidelines on the Diagnosis, Treatment and Isolation of Patients with COVID-19. Journal of Infection Control, Santa Cruz do Sul, RS, v. 9, n. 2, p. 1-20, 2020. Disponível em: http://jic-abih.com.br/index.php/jic/article/view/295. Acesso em: 9 jul. 2020.

DIAZ, A. et al. Elective surgery in the time of COVID-19. American Journal of Surgery, Belle Mead, NJ, v. 219, n. 6, p. 900-902, 2020. DOI: 10.1016/j. amjsurg.2020.04.014. Disponível em: https://www.americanjournalofsurgery.com/ article/S0002-9610(20)30218-X/fulltext. Acesso em: 9 jul. 2020.

BRINDLE, M. E.; GAWANDE, A. Managing COVID-19 in Surgical Systems. Ann Surg, v. 272, n. 1, p. e1-e2, 2020. DOI: 10.1097/SLA.0000000000003923. Disponível em: https://journals.lww.com/annalsofsurgery/FullText/2020/07000/Managing_ COVID_19_in_Surgical_Systems.2.aspx. Acesso em: 9 jul. 2020. 
FERRETTI, L. et al. Quantifying SARS-CoV-2 transmission suggests epidemic control with digital contact tracing. Science, New York, v. 368, n. 6491, 2020. DOI: 10.1126/science.abb6936. Disponível em: https://science.sciencemag.org/ content/368/6491/eabb6936. Acesso em: 9 jul. 2020.

FORRESTER, J. D. et al. Precautions for Operating Room Team Members During the COVID-19 Pandemic. Journal of the American College of Surgeons, Chicago, v. 230, v. 6, p. 1098-1101, 2020. DOI: 10.1016/j.jamcollsurg.2020.03.030. Disponível em: https://www.journalacs.org/article/S1072-7515(20)30303-3/fulltext. Acesso em: 9 jul. 2020.

GALE, S. C. et al. The public health burden of emergency general surgery in the United States: A 10-year analysis of the Nationwide Inpatient Sample--2001 to 2010. Journal of Trauma and Acute Care Surgery, Hagerstown, v. 77, n. 2, p. 202-208, 2014. DOI: 10.1097/TA.0000000000000362. Disponível em: https://journals.lww. com/jtrauma/Abstract/2014/08000/The_public_health_burden_of_emergency_ general.3.aspx. Acesso em: 9 jul. 2020.

GINSBURG, K. B. et al. Delayed Radical Prostatectomy is Not Associated with Adverse Oncological Outcomes: Implications for Men Experiencing Surgical Delay Due to the COVID-19 Pandemic. The Journal of Urology, Baltimore, 2020, DOI: 101097JU0000000000001089. Disponível em: https://www.auajournals.org/ doi/10.1097/JU.0000000000001089. Acesso em: 9 jul. 2020.

GLOSTER JR., H. M.; ROENIGK, R. K. Risk of acquiring human papillomavirus from the plume produced by the carbon dioxide laser in the treatment of warts. Journal of the American Academy of Dermatology, Saint Louis, v. 32, n. 3, p. 436-441, 1995. DOI: 10.1016/0190-9622(95)90065-9. Disponível em: https://www.jaad.org/ article/0190-9622(95)90065-9/pdf. Acesso em: 9 jul. 2020.

HALLAL, P. C. et al. Trends in the prevalence of COVID-19 infection in Rio Grande do Sul, Brazil: repeated serological surveys. Ciência \& Saúde Coletiva, Rio de Janeiro, v. 25, p. 2395-2401, 2020a. Suplemento 1. DOI: https://doi. org/10.1590/1413-81232020256.1.09632020. Disponível em: https://www.scielo. $\mathrm{br} /$ scielo.php?script=sci_arttext\&pid=S1413-81232020006702395\&tlng=pt. Acesso em: 9 jul. 2020.

HALLAL, P. H. et al. Remarkable variability in SARS-CoV-2 antibodies across Brazilian regions: nationwide serological household survey in 27 states. MedRxiv, [s. I.], 2020b. DOI: https://doi.org/10.1101/2020.05.30.20117531. Disponível em: https://www.medrxiv.org/content/10.1101/2020.05.30.20117531v1. Acesso em: 9 jul. 2020. 
HELDWEIN, F. L. et al. A Systematic Review on Guidelines and Recommendations for Urology Standard of Care During the COVID-19 Pandemic. European Urology Focus, Amsterdam, 2020. DOI: 10.1016/j. euf.2020.05.020. Disponível em: https://www.eu-focus.europeanurology.com/ article/S2405-4569(20)30155-3/fulltext. Acesso em: 9 jul. 2020.

HENSMAN, C. et al. Chemical composition of smoke produced by high-frequency electrosurgery in a closed gaseous environment. An in vitro study. Surgical Endoscopy, Berlin, v. 12, n. 8, p. 1017-1019, 1998. DOI: 10.1007/s004649900771. Disponível em: https://link.springer.com/article/10.1007\%2Fs004649900771. Acesso em: 9 jul. 2020.

HORTON, R. Offline: COVID-19 and the NHS-"a national scandal". The Lancet, London, v. 395, n. 10229, p. 1022, 2020. DOI: https://doi.org/10.1016/ S0140-6736(20)30727-3. Disponível em: https://www.thelancet.com/journals/ lancet/article/PIIS0140-6736(20)30727-3/fulltext. Acesso em: 9 jul. 2020.

JOHNSON, G. K.; ROBINSON, W. S. Human immunodeficiency virus-1 (HIV-1) in the vapors of surgical power instruments. Journal of Medical Virology, New York, v. 33, n. 1, p. 47-50, 1991. DOI: 10.1002/jmv.1890330110. Disponível em: https:// onlinelibrary.wiley.com/doi/abs/10.1002/jmv.1890330110. Acesso em: 9 jul. 2020.

$\mathrm{KIM}, \mathrm{H}$. J. et al. Recommendations for anesthesia in patients suspected of COVID-19 Coronavirus infection. Korean Journal of Anesthesiology, Seoul, v. 73, n. 2, p. 89-91, 2020. Disponível em: https://ekja.org/journal/view.php?doi=10.4097/ kja.20110. Acesso em: 9 jul. 2020.

KOVACS, G. et al. Just the Facts: Airway management during the coronavirus disease 2019 (COVID-19) pandemic. CJEM,v. 22, n. 4, p. 440-444. 2020.

DOI: 10.1017/cem.2020.353. Disponível em: https://www.cambridge.org/core/ journals/canadian-journal-of-emergency-medicine/article/just-the-factsairway-management-during-the-coronavirus-disease-2019-covid19-pandemic/ F6386E2DB9FCE5C35EEF313E2210F39E. Acesso em: 9 jul. 2020.

LI, C.-I.; PAI, J.-Y.; CHEN, C.-H. Characterization of smoke generated during the use of surgical knife in laparotomy surgeries. Journal of the Air \& Waste Management Association, Pittsburgh, v. 70, n. 3, p. 324-332, 2020. DOI: https://doi. org/10.1080/10962247.2020.1717675. Disponível em: https://www.tandfonline. com/doi/abs/10.1080/10962247.2020.1717675?journalCode=uawm20.

Acesso em: 9 jul. 2020. 
LIANG, Z. C. et al. COVID-19 and Elective Surgery: 7 Practical Tips for a Safe, Successful and Sustainable Reboot. Annals of Surgery, Philadelphia, 2020. DOI: 10.1097/SLA.0000000000004091. Disponível em: https://journals.Iww.com/ annalsofsurgery/Citation/9000/COVID_19_and_Elective_Surgery_7_Practical_ Tips.94507.aspx. Acesso em: 9 jul. 2020.

LIVINGSTON, E. H. Surgery in a Time of Uncertainty: A Need for Universal Respiratory Precautions in the Operating Room. JAMA, Chicago, v. 23, n. 22, p. 2254-2255, 2020. DOI:10.1001/jama.2020.7903. Disponível em: https:// jamanetwork.com/journals/jama/fullarticle/2765945. Acesso em: 9 jul. 2020.

MAYOR, S. COVID-19: impact on cancer workforce and delivery of care. The Lancet Oncology, London, v. 21, n. 5, p. 633, 2020. DOI: https://doi.org/10.1016/ S1470-2045(20)30240-0. Disponível em: https://www.thelancet.com/journals/ lanonc/article/PIIS1470-2045(20)30240-0/fulltext. Acesso em: 9 jul. 2020.

MOLETTA, L. et al. International guidelines and recommendations for surgery during Covid-19 pandemic: A Systematic Review. International Journal of Surgery, London, v. 79, p. 180-188, 2020. DOI: 10.1016/j.jjsu.2020.05.061. Disponível em: https://www.sciencedirect.com/science/article/pii/ S1743919120304441?via\%3Dihub. Acesso em: 9 jul. 2020.

ONG, S. W. X. et al. Air, Surface Environmental, and Personal Protective Equipment Contamination by Severe Acute Respiratory Syndrome Coronavirus 2 (SARS-CoV-2) From a Symptomatic Patient. JAMA, Chicago, v. 32, n. 16, p. 1610-1612, 2020. DOI:10.1001/jama.2020.3227. Disponível em: https:// jamanetwork.com/journals/jama/fullarticle/2762692. Acesso em: 9 jul. 2020.

PRACHAND, V. N. et al. Medically Necessary, Time-Sensitive Procedures:

Scoring System to Ethically and Efficiently Manage Resource Scarcity and Provider Risk During the COVID-19 Pandemic. Journal of the American College of Surgeons, Chicago, v. 231, n. 2, p. 281-288, 2020. DOI: 10.1016/j.jamcollsurg.2020.04.011. Disponível em: https://www.journalacs.org/article/S1072-7515(20)30317-3/fulltext. Acesso em: 9 jul. 2020.

REMUZZI, A.; REMUZZI, G. COVID-19 and Italy: what next? The Lancet, London, v. 395, n. 10231, p. 1225-1228, 2020. DOI: 10.1016/ S0140-6736(20)30627-9. Disponível em: https://www.thelancet.com/journals/ lancet/article/PIIS0140-6736(20)30627-9/fulltext. Acesso em: 9 jul. 2020. 
ROWLAND, C.; JOHNSON C. Y.; WAN, W. Even finding a covid-19 vaccine won't be enough to end the pandemic. The Washington Post, Washington, 2020. Disponível em: https://www.washingtonpost.com/business/2020/05/11/ coronavirus-vaccine-global-supply/. Acesso em: 9 jul. 2020.

SMITH, W. R. et al. Implementation Guide for Rapid Integration of an Outpatient Telemedicine Program During the COVID-19 Pandemic. Journal of the American College of Surgeons, Chicago, v. 231, n. 2, p. 216-222, 2020. DOI:https:// doi.org/10.1016/j.jamcollsurg.2020.04.030. Disponível em: https://www.journalacs. org/article/S1072-7515(20)30375-6/fulltext. Acesso em: 9 jul. 2020.

SORENSEN, M. J. et al. Telemedicine for Surgical Consultations-- Pandemic Response or Here to Stay? A Report of Public Perceptions. Annals of Surgery, Philadelphia, 2020. DOI: 10.1097/SLA.0000000000004125. Disponível em: https:// journals.Iww.com/annalsofsurgery/Abstract/9000/Telemedicine_for_Surgical_ Consultations_Pandemic.94444.aspx. Acesso em: 9 jul. 2020.

SUD, A. et al. Collateral damage: the impact on outcomes from cancer surgery of the COVID-19 pandemic. Annals of Oncology, Dordrecht, v. 31, n. 8, p. 1065-1074, 2020. DOI: 10.1016/j.annonc.2020.05.009. Disponível em: https://www. annalsofoncology.org/article/S0923-7534(20)39825-2/fulltext. Acesso em: 9 jul. 2020.

ROYAL COLLEGE OF SURGEONS OF EDINBURGH et al. Updated Intercollegiate General Surgery Guidance on COVID-19. [S. I.], 6 Apr. 2020. Disponível em: https:// www.asgbi.org.uk/userfiles/file/covid19/2nd-update-intercollegiate-generalsurgery-guidance-on-covid-19-6-april-_-1.pdf. Acesso em: 9 jul. 2020.

TI, L. K. et al. What we do when a COVID-19 patient needs an operation: operating room preparation and guidance. Canadian Journal of Anaesthesia, New York, v. 6, n. 6, p. 756-758, 2020. DOI: 10.1007/s12630-020-01617-4. Disponível em: https://link.springer.com/ article/10.1007\%2Fs12630-020-01617-4. Acesso em: 9 jul. 2020.

TIAN, Y. et al. Review article: gastrointestinal features in COVID-19 and the possibility of faecal transmission. Alimentary Pharmacology \& Therapeutics, Oxford, v. 51, n. 9, p. 843-851, 2020. DOI: 10.1111/apt.15731. Disponível em: https:// onlinelibrary.wiley.com/doi/full/10.1111/apt.15731. Acesso em: 9 jul. 2020.

VAN DOREMALEN, N. et al. Aerosol and Surface Stability of SARS-CoV-2 as Compared with SARS-CoV-1. The New England Journal of Medicine, Boston, v. 382, n. 16, p. 1564-1567, 2020. DOI: 10.1056/NEJMc2004973. Disponível em: https:// www.nejm.org/doi/10.1056/NEJMc2004973. Acesso em: 9 jul. 2020. 
WANG, W. et al. Detection of SARS-CoV-2 in Different Types of Clinical Specimens. JAMA, Chicago, v. 323, n. 18, p. 1843-1844, 2020. DOI:10.1001/jama.2020.3786. Disponível em: https://jamanetwork.com/journals/jama/fullarticle/2762997. Acesso em: 9 jul. 2020.

WONG, J. et al. Preparing for a COVID-19 pandemic: a review of operating room outbreak response measures in a large tertiary hospital in Singapore. Canadian Journal of Anaesthesia, New York , v. 67, n. 6, p. 732-745, 2020. DOI: 10.1007/s12630-020-01620-9. Disponível em: https://link.springer.com/ article/10.1007\%2Fs12630-020-01620-9. Acesso em: 9 jul. 2020.

$\mathrm{XIAO}, \mathrm{A}$. T. et al. False negative of RT-PCR and prolonged nucleic acid conversion in COVID-19: Rather than recurrence. Journal of Medical Virology, New York, 2020. DOI: 10.1002/jmv.25855. Disponível em: https://onlinelibrary.wiley.com/doi/ full/10.1002/jmv.25855. Acesso em: 9 jul. 2020.

ZHENG, M. H. et al. Minimally Invasive Surgery and the Novel Coronavirus Outbreak: Lessons Learned in China and Italy. Annals of Surgery, Philadelphia, v. 272, n. 1, p. e5-e6, 2020. Disponível em: https://journals.Iww.com/ annalsofsurgery/FullText/2020/07000/Minimally_Invasive_Surgery_and_the_ Novel.4.aspx Acesso em: 9 jul. 2020. 